\title{
PERCEPCIÓN DE LOS GERENTES DE RECURSOS HUMANOS SOBRE HABILIDADES DE LOS CONSULTORES: CONTEXTO MEXICANO
}

\author{
VELASCO LÓPEZ XÓCHITL \\ Universidad Popular Autónoma del Estado de Puebla, México \\ xochitl.velasco@upaep.mx \\ CAVAZOS ARROYO JUDITH \\ Universidad Popular Autónoma del Estado de Puebla, México \\ judith.cavazos@upaep.mx
}

\section{Resumen}

Esta investigación tuvo como propósito identificar la percepción de los gerentes de Recursos Humanos sobre las habilidades de los consultores organizacionales que brindan sus servicios a empresas de un contexto mexicano. Se desarrolló una investigación correlacional y explicativa, con un diseño transversal y la aplicación de una encuesta electrónica a gerentes de Recursos Humanos en la Ciudad de Puebla, México. Los resultados evidenciaron que los sectores empresariales consideran que el consultor debe mejorar en la definición que da el cliente acerca del problema y que la mayor parte de los consultores no tienen algún tipo de certificación. Además, las gerentes de Recursos Humanos consideran que el consultor tiene que desarrollar mejor la habilidad de la valoración de éste sobre el diagnóstico de la empresa. Y los gerentes Senior son más críticos sobre las habilidades generales de los consultores, sobre el sentido y diagnóstico y sobre la negociación de las restricciones y negociaciones del cliente.

Palabras Clave: Gerentes de Recursos Humanos, habilidades de consultoría y Consultor.

\section{Abstract}

This research identifies the perception of $H R$ managers about the skills of organizational consultants who offer their services to companies in a Mexican context. A correlational and explanatory research, with a crossectional design was developed. An electronic survey was applied to HR managers in the city of Puebla, Mexico. The results showed that the business sectors considered that consultants should improve the problem definitions they give to clients, and that most consultants do not have any sort of certification. In addition, female HR managers believe that consultants need to improve their skills when evaluating the diagnosis of the company. Senior managers are more critical about the general skills of consultants, about the meaning of the diagnosis and about negotiations of restrictions and customers.

Keywords: Human Resources Managers, Consulting Skills, Consultan. 


\section{INTRODUCCIÓN}

La consultoría en Recursos Humanos en estos últimos tiempos, se relaciona con la necesidad de respuesta inmediata ante cambios vertiginosos, sin dejar de lado la identidad de la organización ante fenómenos complejos como pueden ser; una fusión, un cambio de cultura organizacional, entre otros. Todo esto, requiere que el consultor cuente con habilidades desde la psicología organizacional que van alineadas con las competencias profesionales, que exigen un agente que sea capaz de identificar los sustentos teóricos de investigaciones y sus efectos en la consultoría psicológica dentro de empresas y; de esta forma, entender las influencias mutuas de los diferentes actores que la integran. De acuerdo a Audirac (2013) la consultoría exige gestionar magistralmente aspectos humanos como satisfacción, actitudes o resistencia al cambio que son propios del desarrollo y la adaptación que se ven reflejados en mejores prácticas de diferentes sectores (Casartelli, 2010).

En México las Pequeñas y Medianas Empresas (PYMES) ocupan el 78.5\% de la fuerza de trabajo, pero son afectadas por el rezago socioeconómico, así como por la cultura empresarial mexicana que tiende a una visión de corto plazo, rechazo a delegar autoridad y responsabilidad y un individualismo que dificulta el trabajo en equipo (Saavedra y Blanca, 2012). Sin embargo, ésta es un área de oportunidad para los consultores ya que permite generar propuestas oportunas e incidir en este sector. La Cámara Nacional de Consultoría (CNC) y la Confederación de Cámaras Industriales de los Estados Unidos Mexicanos (CONCAMIN) concibe a la industria consultora como un medio eficiente y efectivo que puede favorecer la economía mexicana incluyendo a la administración pública (Casartelli, 2010).

De manera más particular, en el estado de Puebla se han desarrollado investigaciones como la de Ramos (2001), quien desde la Consultoría
Psicológica Organizacional propuso la detección de necesidades latentes en 20 pequeñas empresas de diferentes giros afiliadas a la Confederación Patronal de la República Mexicana (COPARMEX) para dar respuesta desde un proceso psicodiagnóstico a la capacitación, eventos recreativos, reclutamiento y selección, motivación de personal, análisis de puestos, comunicación organizacional, administración del tiempo libre, organización y creación del departamento de talento humano y solución a problemas como rotación y ausentismo, entre otros. Por ello, esta investigación tiene como propósito identificar la percepción de los gerentes de Recursos Humanos sobre las habilidades de los consultores organizacionales que brindan sus servicios a empresas de la entidad.

\section{REVISIÓN DE LA LITERATURA}

Aunque la economía mundial no ha tenido un dinámico crecimiento durante los primeros años de la segunda década del siglo XXI, la industria de la gestión del talento se ha mantenido en crecimiento y gran parte de ello proviene tanto de la necesidad de desarrollo organizacional, como de la capacitación (Kaiser y Curphy, 2013). Los consultores organizacionales suelen describirse como especialistas y expertos en la "gestión del cambio", ayudando a las organizaciones a aprender, facilitar el cambio organizacional y que éstas continúen con su desarrollo cuando ellos se van (Berry y Oakley, 1994; Smith, 2002).

Las firmas de consultoría añaden valor a las organizaciones a través de la experiencia, los consejos, las habilidades y la posición externa del consultor, presentando una visión desapasionada de la situación respecto de quienes integran la firma. En este sentido, la percepción que se obtenga tanto de la firma consultora como del consultor son relevantes para la contratación, jugando un rol activo tanto los aspectos profesionales como personales (Berry y Oakley, 1994). Las relaciones consultor-cliente no necesariamente 
están libres de tensiones, sin embargo, se han identificado aspectos críticos que pueden contribuir a fortalecer la relación cliente-consultor (Smith, 2002): 1) alineación de las actividades de consultoría con los objetivos del cliente a través de parámetros claramente comprendidos por ambas partes, 2) la organización cliente involucra stakeholders (públicos interesados en ella), más allá de quienes patrocinan o contratan los servicios del consultor, 3) buena comunicación entre consultor-cliente, 4) producir e implementar las estructuras, tecnología, staff y sistemas necesarios para el cambio organizacional, lo que lleva a coordinar y administrar los recursos que apoyan el proceso, 5) la valoración del desempeño del consultor requiere un entendimiento de cómo los stakeholders, visualizan el desempeño del consultor frente a las expectativas sobre el esfuerzo del cambio.

Las habilidades del consultor van encaminadas esencialmente a la relación que se tiene cliente consultor, así como a la capacidad que tenga el consultor en su manejo de comunicación en entornos profesionales, la habilidad de percepción socio-emocional que se da en la interacción con el cliente, el fomento de relaciones a largo plazo arraigadas en la confianza mutua y un adecuado manejo de los recursos (económicos, materiales, información entre otros) (Plenge, Erickson y Roloff, 2007).

También las habilidades del consultor van encaminadas a cambiar el paradigma que se tiene sobre este servicio, tomando en cuenta que ha sido visto como un servicio que va con la moda, el prejuicio de que el consultor quiere quedarse permanente en la empresa para seguir percibiendo ingresos, la falta de honestidad en el proceso por parte del consultor en donde reconoce sus alcances dentro de la empresa, el consultor se convierte en un instrumento de las políticas organizacionales con tal de satisfacer las necesidades del cliente para incorporarse a una nueva venta, así entre otras ideas que es necesario modificar en las creencias del cliente hacia la consultoría organizacional (Russell, 2001).

Algunas necesidades que se plantean las empresas consultoras pueden ser vistas desde diferentes enfoques, uno de ellos es el de las PYMES que tienden a la ausencia de diversidad de diagnóstico e intervención organizacional, pero cuando requieren los servicios de consultoría propicia una oportunidad para que ésta se actualice en las necesidades y requerimientos de este tipo de clientes, hasta lograr que estos sean independientes (Cohen, 2003).

La consultoría tiene la necesidad de aportar conocimiento, desarrollar técnicas, herramientas y estrategias adecuadas centrándose en las necesidades de cada organización para generar y desarrollar calidad, más que imponer su propia metodología (Marimon, Casadesús y Heras, 2006). También la consultoría tiene que generar aspectos eficientes que la ayuden a posicionarse a través de (Pérez, Barceló, Navarro y Pompa, 2010; Palomo, 2005): precio competitivo, calidad, cobertura de la fuerza de venta y aspectos de publicidad y promoción del producto; a su vez, debe considerar si la capacitación responde a necesidades reales; si existe una cultura de innovación y desarrollo tecnológico y; finalmente, medir el impacto de la gestión en el desarrollo organizacional.

Por otro lado, las unidades de Recursos Humanos han tenido diferentes cambios pasando de un enfoque alineado a lo administrativo, hasta covertirse en un eje estratégico para la organización (López, Sepúlveda y Arenas, 2010). Esto ha generado que el gerente de Recursos Humanos sea más cuidadoso ante la llegada de un consultor, sobretodo en PYMES ya que no cuentan con especialistas a diferencia de las empresas grandes y sus recursos tienden a ser más escasos. La consultoría también es vista por el gerente de Recursos Humanos como una alternativa para conocer sobre cómo se encuentran otras 
empresas, identificar una perspectiva objetiva sobre la situación de la compañía, una oportunidad de mejorar y resolver problemas (Marciano, Mafra y Benetti, 2009).

En una encuesta realizada a 350 gerentes en Recursos Humanos muestra la importancia de que el capital humano, sea un eje estratégico para incidir en la consultoría y la gestión al cambio organizacional, esto puede tener un mejor resultado si se toman en cuenta seis competencias a las cuales el gerente de Recursos Humanos tiene que encaminarse, las cuales son: diseñador del talento organizacional, cultura y cambio, arquitecto de la estrategia, albacea operativo, aliado de negocios y activista creíble (Younger, Younger y Thompson, 2011).

La consultoría efectiva implica el desarrollo de diversas competencias funcionales. Newell, Newell y Looser (2013) consideran cuatro de ellas relevantes: a) la identificación del rol más adecuado a asumir por parte del consultor para una determinada situación, b) la selección de herramientas y procedimientos de evaluación adecuados para abordar el problema, c) proporcionar retroalimentación y recomendaciones efectivas y, d) el uso de la literatura de investigación para la práctica de la consultoría.

\section{METODOLOGÍA}

Se desarrolló una investigación correlacional y explicativa, con un diseño transversal simple y la aplicación de una encuesta electrónica a 22 gerentes de Recursos Humanos en la Ciudad de Puebla, México.

\section{INSTRUMENTO}

Se aplicó una adaptación del "Inventario sobre habilidades de Consultoría" (IHC) (Peralta, 2009). El cuestionario incluyó datos sociodemográficos y cuatro apartados: 1) Habilidades generales $(H G)$, que son los requerimientos esenciales a cubrir por un consultor (13 reactivos), 2) Sentido y diagnóstico (SD), que incluyen los ejes para diagnosticar (10 reactivos), 3) Contratación - Negociación (N) incluyéndose 12 reactivos sobre la capacidad de negociación y, 4) Metodología (M), que evalúa el proceso de consultoría con 15 reactivos. La consistencia interna demostró un alfa de Cronbach de 0.710

\section{RESULTADOS}

\section{CARACTERÍSTICAS DEMOGRÁFICAS}

De los 22 gerentes de Recursos Humanos, 36,4\% (8) laboran en el sector servicios, $13,6 \%$ (3) en la industria automotriz, 9,1\% (2) en el sector educativo y el 40,9\% (9) en otros diversos giros. La mitad de los encuestados fueron del sexo femenino. La mayor parte de los participantes tenían entre 25 y 30 años (40,9\%) seguido por 41 y 50 años (22,7\%), 51 y 60 años (13,6\%), 31 y 35 años $(13,6 \%)$ y entre 36 y 40 años $(9,1 \%)$. El $50 \%$ de los entrevistados contaba con nivel licenciatura, el $40.9 \%$ con maestría, seguido por una especialidad (4,5\%) y doctorado (4,5\%). Finalmente en relación a los años de experiencia laboral 63,6\% tiene entre 1 y 5 años, 27,3\% entre 6 y 10 años y el 9,1\% entre 11 y 15 años (Tabla 1).

\section{ANÁLISIS POR SECTOR EMPRESARIAL}

A través de Anova de un factor (ANOVA) se examinaron las diferencias de los sectores donde laboran los gerentes de Recursos Humanos (RH) $\left(\bar{x}_{1}=\right.$ automotriz, $\bar{x}_{2}=$ educativo, $\bar{x}_{3}=$ servicios, $\bar{x}_{4}=$ otros) respecto de las medias de las percepciones de cada uno de tres de los cuatro apartados de las habilidades de los consultores (habilidades generales, sentido y diagnóstico, contratación y negociación). Asimismo, para la dimensión "metodología", medida en escalas categóricas, se realizó un análisis de tabulación cruzada. Los resultados evidencian que: 
TABLA 1

\section{CARACTERÍSTICAS DEMOGRÁFICAS DE LOS GERENTES DE RECURSOS HUMANOS}

\begin{tabular}{|c|c|c|c|c|c|}
\hline Sector & Frecuencia & $\%$ & sexo & Frecuencia & $\%$ \\
\hline Servicios & 8 & 36.4 & Femenino & 11 & 50 \\
\hline Automotriz & 3 & 13.6 & Masculino & 11 & 50 \\
\hline Educativo & 2 & 9.1 & $n$ & & \\
\hline Otros & 9 & 40.9 & & & \\
\hline$n$ & 22 & 100 & & & \\
\hline Edad & Frecuencia & $\%$ & Escolaridad & Frecuencia & $\%$ \\
\hline $25-30$ & 9 & 40.9 & Licenciatura & 11 & 50 \\
\hline $31-35$ & 3 & 13.6 & Maestría & 9 & 40.9 \\
\hline $36-40$ & 2 & 9.1 & Especialidad & 1 & 4.5 \\
\hline $41-50$ & 5 & 22.7 & Doctorado & 1 & 4.5 \\
\hline $51-60$ & 3 & 13.6 & $n$ & 22 & \\
\hline$n$ & 22 & & & & \\
\hline Años de experiencia & Frecuencia & $\%$ & & & \\
\hline 1-5 años & 14 & 63.6 & & & \\
\hline 6-10 años & 6 & 27.3 & & & \\
\hline 11-15 años & 2 & 9.1 & & & \\
\hline$n$ & 22 & 100 & & & \\
\hline
\end{tabular}

Fuente: Elaboración propia.

Habilidades generales. Ninguna de las habilidades generales presenta diferencias significativas entre las percepciones de los gerentes de RH según los sectores atendidos.

Sentido y diagnóstico. Solamente la percepción de que la empresa valorará que el consultor acepta la definición que da el cliente acerca del problema resultó marginalmente significativa ( $p=0.057$ ) resultando más positiva en el sector educativo $\left(\bar{x}_{2}=1.50\right)$, seguido del sector automotriz $\left(\bar{x}_{1}=1.33\right)$, otros giros $\left(\bar{x}_{4}=.78\right)$ y por último el sector servicios ( $\bar{x}_{3}=.25$ ). Lo anterior implica que el sector educativo considera que el consultor debe aceptar menos la definición que da el cliente acerca del problema, el sector automotríz y otros giros perciben que el consultor necesita desempeñarse más en la definición que da el cliente del problema y finalmente el sector servicios consideran que se hace correctamente.

Contratando y negociando. Ninguna de las variables presenta diferencias significativas entre las percepciones sobre el consultor respecto a los diferentes sectores.

Metodología. Los resultados evidenciaron que ninguna de las variables de este rubro se asocia con respecto de los giros incluidos en el estudio. La mayor parte de los giros sostienen que el consultor no tiene algún tipo de certificación, que el consultor para incursionar en el ámbito de la consultoría debe conocer principalmente sobre $\mathrm{RH}$ y después sobre aspectos administrativos. 


\section{ANÁLISIS POR GÉNERO}

Se realizó una prueba T para muestras independientes por género, respecto de las medias de las percepciones de habilidades generales, sentido y diagnóstico y contratación - negociación. Para la dimensión de metodología se utilizó un análisis de tabulación cruzada. Los resultados reflejan que:

Habilidades generales. Se encontró una diferencia significativa respecto a la percepción entre hombres (1) y mujeres (2) en relación a la valoración del consultor sobre lo que tiene la empresa ( $p=.003$ ). Los resultados evidencian que las gerentes de $\mathrm{RH}$ consideran que su consultor necesita desempeñarse mejor en relación a esta habilidad, en cambio los gerentes hombres de RH valoran positivamente esta habilidad en el consultor considerando que se hace correctamente $\left(\bar{x}_{1}=.27, \bar{x}_{2}=1.18\right)$.

Sentido y diagnóstico. No se encontraron diferencias significativas respecto a las percepciones entre hombres (1) y mujeres (2) en relación a las nueve variables de esta dimensión. La variable mejor evaluada se relaciona con la habilidad del consultor para hacer preguntas directas, pero ambos sexos consideran que se debe desempeñar más que la empresa valore que el consultor espere a que los clientes utilicen las soluciones.

Contratación - negociación. No se identificaror diferencias significativas respecto a las percepciones entre gerentes hombres (1) y gerentes mujeres (2) en relación a las variables de esta dimensión. Lo que se percibe que se hace más correctamente por parte de los consultores desde el punto de vista de los gerentes de RH es la negociación sobre los honorarios y la que necesita desempeñarse más es la adaptación del consultor para aceptar las restricciones y limitaciones del cliente.
Metodología: Los resultados no evidencian asociación entre género y los aspectos metodológicos del consultor respecto de las variables analizadas.

\section{ANÁLISIS POR EDAD}

A través de un Anova de un factor se examinaron las diferencias entre cinco grupos de edad identificados ( $1=25$ a 30 años, $2=31$ a 35 años, $3=36$ a 40 años, $4=41$ a 50 años, $5=51$ a 60 años) respecto a las distintas habilidades generales, el sentido y diagnóstico, contratación y negociación y metodología utilizada.

Habilidades generales. Los gerentes de RH piensan que la empresa valorará la habilidad del consultor para ser breve y conciso ( $p=.037)$. La prueba pos hoc HSD de Tukey evidencia que las diferencias se perciben entre los gerentes más jóvenes, quienes piensan que esta habilidad la desempeña correctamente el consultor, y los gerentes entre 31 y 35 años quienes piensan que el consultor de su organización necesita desempeñar más esta habilidad.

Sentido y diagnóstico. El análisis evidencia solamente una diferencia significativa respecto a la percepción de que la empresa valorará que el consultor ayude a los clientes a descubrir sus propios problemas ( $p=$.032). La prueba pos hoc HSD de Tukey evidencia que el grupo de edad entre 36 y 40 años consideran que se desempeña correctamente esta tarea mientras que los gerentes entre 31 y 35 piensan que se debe desempeñar más el consultor en esta tarea.

Contratación - negociación. Una variable fue significativa ( $p=.021)$, la cual implica que los gerentes más jóvenes perciben que el consultor se desempeña bien aceptando las restricciones y limitaciones del cliente. Sin embargo los gerentes entre 41 y 50 años son los más críticos sosteniendo que debe desempeñarse mejor esta competencia. 
Metodología: Se identificó una asociación entre edad y labor de consultoría independiente del trabajo por parte de los gerentes de RH ( $p=.043)$. Los resultados muestran que los gerentes entre 31 y 50 años son los que más se dedican solamente a su desempeño dentro de la empresa pero, los gerentes más jóvenes (25 - 30) y los gerentes con más experiencia (51 - 60) manifiestan realizar labores de consultoría de forma adicional a su desempeño dentro de la organización donde laboran.

\section{DISCUSIÓN}

Los resultados evidenciaron que los gerentes de recursos humanos perciben que es necesario que el consultor valore más lo que la empresa tiene. Dos elementos clave de interacción en el proceso de consultoría se encuentran en la formulación del problema y la identificación de oportunidades, ya que las percepciones y sesgos sobre estos aspectos incidirán en la toma de decisiones durante el proceso de cambio (Thomas y Schwenk, 1983; Kumar, Simon y Kimberley, 2000). Los colaboradores tienen percepciones y conexiones respecto a la organización en la que laboran y éstas pueden diferir de las del consultor, por tanto una forma de generar mayor valor en el proceso de consultoría es a través de la creación y gestión del conocimiento existente, tanto explícito como tácito, éste último en poder y uso de los colaboradores de la organización (Lu y Sexton, 2006). Dentro de los activos organizacionales más valiosos se encuentra el capital humano, el cual contribuye desde sus capacidades, habilidades y gestión en la creación de valor, incluso como consultor interno puede reconocer de forma más precisa y profunda la situación y contexto de un problema organizacional (Simon, 2007).

Ninguno de los gerentes de Recursos Humanos de los sectores productivos estudiados percibió diferencias respecto a las habilidades generales de los consultores. El agente de cambio es valorado en las organizaciones por su capacidad de promover acciones innovadoras que permitan anticiparse a las nuevas situaciones de las organizaciones. Además es un gestor de la cultura organizativa, cuya tarea implica no sólo el diagnosticar y conocer la cultura existente en la organización, sino el diseminarla a través de las orientaciones dadas a las prácticas de gestión de los recursos humanos (Simon, 2007).

Por otro lado, los gerentes de Recursos Humanos sostienen que los consultores no tienen algún tipo de certificación. La Cámara Nacional de Empresas de Consultoría (2009) sostiene que además de que el consultor debe ser un profesional calificado, actualizado en su especialidad o especialidades, debe contar con la experiencia necesaria para insertarse en este ámbito, ya que su imagen puede ser respaldada mediante una certificación. La obtención de una certificación es un factor clave para la generación de una ventaja competitiva respaldando los servicios y habilidades del consultor (Valencia, 2008).

Se encontró que las percepciones respecto a las habilidades generales de los consultores difieren entre gerentes masculinos y femeninos, específicamente en relación a la capacidad del consultor para valorar la empresa. Cohn (2000) menciona que las gerentes mujeres presentan visibilidad aumentada en las organizaciones, por lo que tienen más presión para llevar a cabo tareas y proyectos con éxito. Por su parte Watts (2009), sostiene que las mujeres gerentes tienen que legitimar más su estilo de gestión y se encuentran bajo más escrutinio organizacional por tanto, deben demostrar más eficientemente su rol como gestoras. Por ejemplo, Brush y Bird (1996) argumentan que las gerentes son menos dependientes de estrategias formalizadas a largo plazo y buscan más la innovación y la flexibilidad, es más probable que las mujeres busquen la participación, estén más orientadas a la consulta y la comunicación (Dainty et al., 2006; Carli y Eagly, 
2007). Por lo anterior, es posible afirmar que las directoras de Recursos Humanos requieran que el consultor perfeccione su habilidad sobre los mecanismos y herramientas para valorar la empresa. Las mujeres tienden a preocuparse por objetivos más amplios vinculando el éxito global de la empresa a su propio éxito profesional y viceversa (Debeljuh y Las Heras, 2013).

Los resultados por rango de edad muestran que en general los gerentes de Recursos Humanos con más experiencia valoran que el consultor sea breve, conciso y negocie asertivamente las restricciones y limitaciones del cliente. Estos resultados coinciden con los de Pellegrinelli (2002), quien sostiene que habilidades como éstas se ven influidas también por la relación que se ha construido entre cliente y consultor. Por su parte Ramírez (2009), considera que el consultor debe ser asertivo al analizar los riesgos potenciales y los beneficios asociados con los cursos de acción. Los consultores junior tienden a aceptar más las restricciones y limitaciones de los clientes, en cambio los consultores senior son visualizados como más eficaces y eficientes al realizar las negociaciones, agregando valor al proceso de consultoría (González, Rodríguez y Duarte, 2011).

\section{CONCLUSIONES}

Esta investigación identificó las percepciones que los gerentes de Recursos Humanos tienen sobre las habilidades de los consultores organizacionales que brindan sus servicios a empresas de la entidad estudiada. Los consultores pueden ser evaluados por los gerentes de Recursos Humanos desde diferentes dimensiones. En esta investigación se incluyeron cuatro: habilidades generales, sentido y diagnóstico, contrataciónnegociación y metodología del consultor. Estos aspectos fueron analizados a través de tres características demográficas, sector empresarial, sexo y edad de los gerentes.
Respecto a los sectores empresariales estudiados, destacan dos percepciones encontradas respecto al sentido y diagnóstico del consultor al intervenir en la organización, ya que se encontró que el sector educativo considera que el consultor debe indagar más sobre las causas de la problemática de la institución, más que guiarse solamente por lo que el cliente exprese. Mientras que los gerentes de la industria automotriz puntualizan la necesidad de que el consultor otorgue más prioridad a las solicitudes del cliente. Por otro lado, los giros concuerdan en que la mayor parte de los consultores no tienen algún tipo de certificación, un aspecto que puede convertirse en un valor agregado para la consultoría y que merece atención para futuras investigaciones.

Por otro lado, la percepción según el sexo de los(as) gerentes de Recursos Humanos mostró que las gerentes son más críticas al evaluar las habilidades de consultoría organizacional, ya que consideran de acuerdo a su experiencia de intervenciones anteriores, que el consultor tiene que desarrollar mejor la habilidad de la valoración sobre el diagnóstico de la empresa, mientras que los hombres califican mejor dicha habilidad. Una posible explicación de esta diferencia puede sustentarse en que las mujeres gerentes sienten que deben demostrar su eficiencia en la gestión, por lo que podrían exigir más respecto de la calidad de los servicios que contratan y están bajo su responsabilidad.

Finalmente, respecto a la edad los gerentes Senior son más críticos sobre las habilidades generales de los consultores, sobre el sentido y diagnóstico y sobre la negociación de las restricciones y del cliente. La experiencia de intervenciones anteriores, la madurez de una relación cliente-consultor y otros aspectos psico-sociales pueden incidir en la adquisición de un enfoque sistémico que permite tanto al gerente como al consultor obtener una visión holística de las fortalezas y áreas de oportunidad de la organización ante un proceso de cambio. Los resultados de este estudio 
contribuyen a la comprensión de los servicios de consultoría organizacional en México, un campo aún poco explorado en el país. No obstante, las limitaciones del estudio involucran que un número relativamente reducido de gerentes de Recursos Humanos participaron en la investigación y el alcance espacial solamente comprende al estado de Puebla, por lo que estudios futuros pueden ser aplicados en otros contextos del país y Latinoamérica, utilizándose muestras más amplias. Consideramos que el consultor debe encaminarse a construir una relación asertiva con el departamento de Recursos Humanos para generar valor agregado a sus servicios profesionales. Algunas herramientas evidenciadas en esta investigación implican la certificación de sus habilidades, buscando la co-participación para un mejor diagnóstico y solución de problemas así como, la mejora de la comunicación con los directores de este departamento.

\section{REFERENCIAS}

Audirac, C. (2013). Desarrollo organizacional y consultoría (1a edición ed.). México: Trillas.

Berry, A. and Oakley, K. (1994). Consultancies: agents of organizational development. Part II. Leadership \& Organization Development Journal, 15(1), 13-21.

Brush, C. G. and Bird, B. J. (1996) Leadership vision of successful women entrepreneurs: dimensions and characteristics, in W. D. Bygrave, B. J. Bird, S. Birley, N. C. Churchill, M. G. Hay, R. H. Kelley and W. E. Wetzel, Jr (Eds) Frontiers of Entrepreneurship Research, Summary. Miami: Center for Entrepreneurial Studies, Babson College, Babson Park.

Cámara Nacional de Empresas de Consultoría (CNEC) (2009). Recomendaciones para elaborar propuesta de servicios de consultoría. Secretaria de Comunicaciones y transportes. México. Recuperado de: http://www.sct.gob.mx/ fileadmin/DireccionesGrales/DGTFM/Licitaciones/2010/9082_01_10/03_Anexo_C_2010.pdf
Carli, L. L. and Eagly, A. H. (2007) Overcoming resistance to women leaders: the importance of leadership style in B. Kellerman and D. L. Rhode (eds.) Women \& Leadership (pp. 127-148). San Fransisco: John Wiley.

Casartelli, G., (2010). Desarrollo de la Industria Consultora de México: Direcciones Estratégicas, Agenda de Acciones y Política. Inter-American Development Bank. No. IDB-TN-215

Cohen, W. (2003). Cómo ser un consultor exitoso. México: Norma.

Cohn, S. (2000) Race, Gender and Discrimination at Work. Colorado, USA: Westview Press.

Dainty, A., Moore, D., \& Murray, M. (2007). Communication in construction: Theory and practice. Routledge. London and New York: Taylor y Francis Group.

De Faria., A. (2004). Desarrollo organizacional: enfoque integral. México: Limusa.

Debeljuh, P. y Las Heras, M. (2013). Mujer y liderazgo. México: IPADE. LIB.

González-Millán, J. J., Rodríguez-Díaz, M. T. y Duarte, L. M. (2011). Necesidades de consultoría en las MIPYMES del Valle de Sugamuxi en Boyacá. Cuadernos de Administración, 27(46) 67-80. Recuperado de: http://www.redalyc.org/articulo. oa? id $=225022711006$

Kaiser, R. B. and Curphy, G. (2013). Leadership development: The failure of an industry and the opportunity for consulting psychologists. Consulting Psychology Journal: Practice and Research, 65(4), 294-302.

Kumar, V., Simon, A. and Kimberley, N. (2000). Strategic capabilities which lead to management consulting success in Australia. Management Decision, 38(1), 24-35.

López, E., Sepúlveda, C. I. y Arenas, H. A. (2010). La consultoría de gestión humana en empresas medianas. Estudios Gerenciales, 26(114) 149-168. Recuperado de: http://www.redalyc.org/articulo. oa?id=21218572007 
Lu, S. and Sexton, M. (2006). Innovation in small construction knowledge-intensive professional service firms: a case study of an architectural practice. Construction Management and Economics, 24 (12), 1269-1282.

Marciano, D., Mafra, E. and Benetti K. C. (2009). A Consultoria Interna de Recursos Humanos como Prática Catalisadora da Gestão do Conhecimento Organizacional. Revista de Ciências da Administração,, 11 (25), 151-176.

Marimon, F. Casadesús, F. and Heras, I. (2006). ¿Consulta o inculcación de principios? Universia Business, 9, 74 - 87.

Newell, M., Newell, T. and Looser, J. (2013). Examining how novice consultants address cultural factors during consultation: illustration of a computer- simulated case-study method. Consulting Psychology Journal, 65 (1) 74-86. http://dx.doi. org/10.1037/a0032598

Palomo, G. (2005). Los procesos de gestión y la problemática de las PYMES. Ingenierías, 8 (28), 1 - 7.

Pellegrinelli, S. (2002). Managing the interplay and tensions of consulting interventions: The consultant-client relationship as mediation and reconciliation. Journal of Management Development, 21(5), 343-365.

Peralta, S. (2009). Consultoría de Sistemas: una forma de resolver problemas organizacionales. (C.d. organizacionales, Productor) Recuperado de: www. universidaddesancarlosdeguatemala.com

Pérez, A., Barceló, M., Navarro, R. y Pompa, F. (2010). Impacto de la Consultoría en el Noroeste de México por parte de las PyMeES- TI. Sistemas, Cibernética E Informática, 2 (2) 61-65

Plenge, N., Erickson, R. and Roloff M. (2007). The influence of situational constraints on the perceived value of consulting services. International Journal of Organizational Analysis, 15(2), 136-151.
Ramírez, L. (2009). Los dilemas éticos que enfrenta el/ la psicólogo/a en su rol de consultor/a al intervenir en un proceso de desplazamiento de empleados/as. Revista Puertorriqueña de Psicología, 20, 47-58. Recuperado de http://www.redalyc. org/articulo.oa?id=233216361003

Russell, W. (2001). The client's role in the consulting relationship: is there "con" in consulting? Managerial Auditing Journal, 16 (9), 519-522. http:// dx.doi.org/10.1108/EUM0000000006084

Saavedra, G. y Blanca T., (2012). El entorno sociocultural y la competitividad de la PYME en México. Panorama socioeconómico, 30 (44), 4 - 24.

Simon, D. (2007). La gestión de los Recursos Humanos. México: McGrawHill.

Smith, M. E. (2002). What client employees say about consultants. Leadership \& Organization Development Journal, 23(2), 93-103.

Thomas, H. and Schwenk, C. (1983). Problem formulation and the consultant-client relationship. Interfaces, 13(5), 25-34.

Valencia, P. (2008). Tesis inédita de trabajo de grado práctica empresarial: Apoyo al área de consultoría en recursos humanos de human perspectives international, inc - Colombia. Universidad Pontificia Bolivariana, Bucaramanga.

Watts, J. H. (2009). Leaders of men: women 'managing' in construction. Work, Employment and Society, 23(3), pp. 512-530.

Younger, J., Younger, A. and Thompson, N. (2011). Developing the skills of HR business partnership: consulting and change management. Strategic HR Review, 10 (1), 6-14. http://dx.doi. org/10.1108/14754391111091751

Recibido: 26 de diciembre de 2015 Aceptado: 13 de marzo de 2016 
This document was created with Win2PDF available at http://www.win2pdf.com. The unregistered version of Win2PDF is for evaluation or non-commercial use only. This page will not be added after purchasing Win2PDF. 